Pacific Journal of Mathematics

THE SCHWARZIAN DERIVATIVE AND THE POINCARE JACOB BURBlE 


\title{
THE SCHWARZIAN DERIVATIVE AND THE POINCARÉ METRIC
}

\author{
JACOB BURBEA \\ Dedicated to Z. Nehari
}

Let $\Omega \notin 0_{G}$ be a plane region and let $\lambda_{\Omega}(z)$ be its Poincaré metric. Let $E_{\Omega}$ be the complement of $\bar{\Omega}$ and write $\alpha(\zeta)=\alpha(\zeta: \Omega)=$ $\left\{\pi^{-1} \int_{E_{\Omega}}|z-\zeta|^{-4} d \sigma(z)\right\}^{1 / 2}$, where $d \sigma(z)=d x d y$ and $\zeta \in \Omega$. $\lambda_{\Omega}(z) \stackrel{E}{=} \alpha(z: \Omega)$ for all $z \in \Omega$ only when $\Omega$ is a disk less (possibly) a closed subset of inner capacity zero. Let $\phi$ be holomorphic and univalent in $\Omega$ and let $S_{\phi}(z, \zeta)=-6\left(\partial^{2} / \partial z \partial \zeta\right) \times$ $\log (\phi(z)-\phi(\zeta)) /(z-\zeta)$. Here $S_{\phi}(z, z)$ is the Schwarzian derivative of $\phi$. We show

$$
\left|S_{\phi}(z, \zeta)\right| \leqq 6 \lambda_{\Omega}(z) \lambda_{\Omega}(\zeta)\left[1+\left(1-\frac{\alpha^{2}(\zeta)}{\lambda_{\Omega}^{2}(\zeta)}\right)^{1 / 2}\right] ; \quad z, \zeta \in \Omega
$$

1. Introduction. In his paper [4] Gehring was concerned with the problem of extending to an arbitrary simply connected plane region $\Omega$ certain results relating the univalence of a function $\dot{\phi}$ holomorphic in the unit disk $\Delta$ with the magnitude of its Schwarzian derivative

$$
S_{\phi}(z)=\left(\frac{\phi^{\prime \prime}}{\dot{\phi}^{\prime}}\right)^{\prime}-\frac{1}{2}\left(\frac{\dot{\phi}^{\prime \prime}}{\dot{\phi}^{\prime}}\right)^{2} ; \quad \phi=\phi(z), z \in \Delta .
$$

We shall be concerned with generalizing the following two propositions to an arbitrary plane region $\Omega$.

Proposition 1. If $\dot{\phi}$ is holomorphic and univalent in $\Delta$, then

$$
\left|S_{\phi}(z)\right| \leqq 6\left(1-|z|^{2}\right)^{-2}, \quad z \in \Delta,
$$

and the constant 6 is sharp.

Proposition 2. Let $\Omega$ be a simply connected domain and let $\lambda_{\Omega}(z)$ be its Poincaré metric. If $\dot{\phi}$ is holomorphic and univalent in $\Omega$, then

$$
\left|S_{\phi}(z)\right| \leqq 12 \lambda_{\Omega}^{2}(z), \quad z \in \Omega,
$$

and the constant 12 is sharp.

Proposition 1 is due to Kraus [5] and Proposition 2 is due to Lehto [6]. In this direction Nehari [7] has shown that if $\phi$ is holomorphic with $\left|S_{\phi}(z)\right| \leqq 2\left(1-|z|^{2}\right)^{-2}$ in $\Delta$, then $\phi$ is univalent in $\Delta$ with the constant 2 being the best possible. 
We shall show that the above two propositions can be immediately read off from one single inequality (Corollary 3) which is valid for any plane region. Our result can be easily extended to open Riemann surfaces too but we shall not pursue this point. Our arguments rely heavily on well known classical results of Bergman and Schiffer [2]. In order to be self contained, however, we will attempt to provide proofs to most crucial statements. The final result obtained in this paper (Theorem 2) involves a string of sharp inequalities amongst the Schwarzian derivative, the span or condenser capacity, the analytic capacity, the capacity and the Poincaré metric. In this string of inequalities, the inequality between the span (condenser) capacity and the analytic capacity is a well known result of Ahlfors and Beurling [1]. Here we provide a different proof of this result which is based on our Theorem 1. The representation formula of Theorem 1 was first mentioned in Schiffer [9] in case $\zeta=\infty \in \Omega$ and $\Omega$ has the largest complementary area amongst all regions which are conformally equivalent to $\Omega$.

2. Capacities and the Poincaré metric. Let $\Omega$ be an open region in the extended plane and let $\zeta \in \Omega$. Usually, $\zeta \neq \infty$ but the transition to $\zeta=\infty$ is trivial. $H(\Omega)$ stands for the class of holomorphic functions in $\Omega$ and $H_{m}(\Omega)$ denotes the class of multivalued holomorphic functions $f$ in $\Omega$ such that $|f(z)|, z \in \Omega$, is single valued. We write

$$
\|f\|_{\infty}=\sup _{z \in \Omega}|f(z)|, \quad D[f]=\int_{\Omega}\left|f^{\prime}(z)\right|^{2} d \sigma(z),
$$

where $d \sigma(z)=d x d y$ is the Lebesgue area measure. Consider the following families:

$$
\begin{aligned}
& \mathscr{P}_{\zeta}(\Omega)=\left\{f \in H(\Omega):\|f\|_{\infty} \leqq 1, f(\zeta)=0\right\}, \\
& \mathscr{C}_{\zeta}(\Omega)=\left\{f \in H_{m}(\Omega):\|f\|_{\infty} \leqq 1, f(\zeta)=0\right\}, \\
& \mathscr{D}_{\zeta}(\Omega)=\{f \in H(\Omega): D[f] \leqq \pi, f(\zeta)=0\} .
\end{aligned}
$$

We now introduce (cf. [1]) the analytic capacity

$$
C_{B}(\zeta)=C_{B}(\zeta: \Omega)=\max \left\{\left|f^{\prime}(\zeta)\right|: f \in \mathscr{B}_{\zeta}(\Omega)\right\},
$$

the capacity

$$
C_{\beta}(\zeta)=C_{\beta}(\zeta: \Omega)=\max \left\{\left|f^{\prime}(\zeta)\right|: f \in \mathscr{C}_{\zeta}(\Omega)\right\}
$$

and the span or condenser capacity

$$
C_{D}(\zeta)=C_{D}(\zeta: \Omega)=\max \left\{\left|f^{\prime}(\zeta)\right|: f \in \mathscr{D}_{\zeta}(\Omega)\right\}
$$


We note that $C_{\beta}(\zeta)$ is well defined and that by $f(\zeta)=0$ in $\mathscr{C}_{\zeta}(\Omega)$ we mean that at least one branch of $f(z)$ vanishes at $\zeta$.

Assume now that $\Omega \notin 0_{G}$ (i.e., $\Omega$ has a nontrivial Green's function) and thus $\Omega$ has the unit disk $\Delta$ as its universal covering space. The Poincaré metric $\lambda_{\Omega}(z)$ is defined as follows: For the unit disk $\Delta, \lambda_{\Delta}(\omega)=\left(1-|\omega|^{2}\right)^{-1}$ while for $\Omega$

$$
\lambda_{\Omega}(z)=\lambda_{\Delta}(\omega)\left|\pi^{\prime}(\omega)\right|^{-1}, \quad z=\pi(\omega) \in \Omega,
$$

where $\pi: \Delta \rightarrow \Omega$ is a universal cover map. We denote by $\delta_{\Omega}(z)$ the distance from $z$ to the boundary of $\Omega . \quad \lambda_{\Omega}(z)$ is monotonic decreasing with $\Omega$ and thus

$$
\lambda_{\Omega}(z) \delta_{\Omega}(z) \leqq 1, \quad z \in \Omega .
$$

Moreover, if $\Omega$ is simply connected then, in view of Koebe's $1 / 4$ theorem,

$$
\lambda_{\Omega}(z) \delta_{\Omega}(z) \geqq 1 / 4, \quad z \in \Omega .
$$

Clearly, $C_{B}, C_{\beta}, C_{D}$ and $\lambda_{\Omega}$ are conformally invariant and therefore

$$
C_{D}(z: \Omega)=C_{B}(z: \Omega)=C_{\beta}(z: \Omega)=\lambda_{\Omega}(z), \quad z \in \Omega,
$$

whenever $\Omega$ is simply connected.

It is also evident that $C_{B}(z: \Omega) \leqq C_{\beta}(z: \Omega)$ and it is a theorem of Ahlfors and Beurling [1] (see also Corollary 2) that $C_{D}(z: \Omega) \leqq C_{B}(z: \Omega)$. Moreover, $C_{\beta}(z ; \Omega) \leqq \lambda_{\Omega}$. Indeed, let $\pi ; \Delta \rightarrow \Omega$ be a universal cover map $z=\pi(\omega)$. Then

$$
\begin{aligned}
C_{\beta}(z: \Omega) & =\max \left\{\left|f^{\prime}(z)\right|: f \in \mathscr{C}_{z}(\Omega)\right\} \\
& =\left|\pi^{\prime}(\omega)\right|^{-1} \max \left\{\left|g^{\prime}(\omega)\right|: g=f \circ \pi \in \mathscr{C}_{\omega}(\Delta), f \in \mathscr{C}_{z}(\Omega)\right\} \\
& \leqq\left|\pi^{\prime}(\omega)\right|^{-1} \max \left\{\left|g^{\prime}(\omega)\right|: g \in \mathscr{C}_{\omega}(\Delta)\right\} \\
& =\left|\pi^{\prime}(\omega)\right|^{-1} C_{\beta}(\omega: \Delta) \\
& =\lambda_{\Delta}(\omega)\left|\pi^{\prime}(\omega)\right|^{-1}=\lambda_{\Omega}(z),
\end{aligned}
$$

where (2.1) has been used. Consequently,

$$
C_{D}(z: \Omega) \leqq C_{B}(z: \Omega) \leqq C_{\beta}(z: \Omega) \leqq \lambda_{\lessdot c}(z) \leqq \delta_{\Omega}^{-1}(z),
$$

and we note that, if $\Omega \notin 0_{A D}$, then $C_{D}(z: \Omega)>0$ for all $z \in \Omega$. The condition $\Omega \notin 0_{A D}$ means that there exists a nonconstant holomorphic function $f$ in $\Omega$ with $D[f]<\infty$.

We conclude this section by recalling the following well-known lemma of Ahlfors and Beurling [1]:

Lemma 1. Let $E$ be a measurable set with a finite Lebesgue measure $\sigma(E)$ in $C$. Then 


$$
\sup _{\zeta \in \boldsymbol{C}}\left|\int_{E} \frac{d \sigma(z)}{z-\zeta}\right| \leqq \sqrt{\pi \sigma(E)}
$$

with equality holding, if and only if $E$ is a almost everywhere a disk of radius $\sqrt{\sigma(E) / \pi}$.

3. The slit mappings. Here we assume that the region $\Omega$ is bounded by $n$ closed analytic curves $C_{1}, \cdots, C_{n}$ and we denote by $C$ the boundary $\partial \Omega=\bigcup_{k=1}^{n} C_{k}$ of $\Omega$. We assume that $C_{1}$ is outer and we let $\zeta \in \Omega$. Let

$$
p(z)=p(z: \zeta)=\frac{1}{z-\zeta}+a(z-\zeta)+\cdots
$$

and

$$
q(z)=q(z: \zeta)=\frac{1}{z-\zeta}+b(z-\zeta)+\cdots
$$

be the horizontal and vertical slit mappings, respectively, of $\Omega$. We write

$$
\Phi(z)=\Phi(z: \zeta)=\frac{1}{2}(p(z)-q(z))
$$

and

$$
\Psi(z)=\Psi(z: \zeta)=\frac{1}{2}(p(z)+q(z)) .
$$

Then $\Phi(z)$ and $(z-\zeta) \Psi(z)$ are holomorphic on $\bar{\Omega}$ with $\Phi(\zeta)=0$. Further, $\Psi(z)$ is univalent on $\Omega$ with pole at $\zeta$. It maps $\Omega$ onto $\Omega^{*}$ with $E=\hat{C}-\bar{\Omega}^{*}$ being bounded. Clearly, $d \Phi=d \bar{\Psi}$ on $C$ and therefore $\Phi=\bar{\Psi}-\bar{\lambda}_{k}$ on $C_{k}$, where $\lambda_{k}$ is a constant depending on the component $C_{k}, 1 \leqq k \leqq n$. Also $\Phi\left(C_{k}\right)$ and $\Psi\left(C_{k}\right)$ are closed analytic and convex curves. One easily shows that

$$
D[\dot{\phi}]=\pi C_{\nu}^{2}(\zeta: \Omega)=\sigma(E)=\frac{\pi}{2}(a-b) .
$$

Let

$$
\psi(z)=\psi(z: \zeta)=\zeta+\frac{1}{\Psi(z: \zeta)} .
$$

$\psi$ maps $\Omega$ conformally onto $\Omega^{\prime}=\psi(\Omega)$ with $\phi(\zeta)=\zeta$. We write $\Gamma=\bigcup_{k=1}^{n} \Gamma_{k}=\partial \Omega^{\prime}$ with $\Gamma_{k}=\psi\left(C_{k}\right), 1 \leqq k \leqq n$. We now establish an integral formula representing $\Phi$ in terms of $\Psi$ (compare also Schiffer [9]). 
THEOREM 1. The integral formula

$$
\Phi(z: \zeta)=\frac{1}{\pi} \int_{E} \frac{d \sigma(t)}{\Psi(z: \zeta)-t} ; \quad E=\hat{\boldsymbol{C}}-\overline{\Psi(\Omega)},
$$

holds.

Proof. We first note that if $f$ is holomorphic on $\bar{\Omega}$ then, using the residue theorem,

$$
f(z)=\frac{1}{2 \pi i} \int_{C} f(\tau) \frac{\psi^{\prime}(\tau)}{\psi(\tau)-\psi(z)} d \tau
$$

Specializing this formula for $f=\Phi \Psi$ we obtain

$$
\Phi(z) \Psi(z)=\frac{1}{2 \pi i} \int_{C}|\Psi(\tau)|^{2} \frac{\psi^{\prime}(\tau)}{\psi(\tau)-\psi(z)} d \tau-\frac{1}{2 \pi i} \sum_{k=1}^{n} \bar{\lambda}_{k} \int_{c_{k}} \psi(\tau) \frac{\psi^{\prime}(\tau)}{\psi(\tau)-\psi(z)} d \tau
$$

Writing $w=\psi(z)$ and $\omega=\psi(\tau), \tau=C$, and recalling the definition of $\psi(z)=\psi(z: \zeta)$ we have

$$
\int_{C_{k}} \psi(\tau) \frac{\psi^{\prime}(\tau)}{\psi(\tau)-\psi(z)} d \tau=\int_{\Gamma_{k}} \frac{1}{\omega-\zeta} \frac{1}{\omega-w} d \omega
$$

which is zero for each $k=1, \cdots, n$. Therefore, writing $f=\Phi \Psi$,

$$
f(z)=\frac{1}{2 \pi i} \int_{\Gamma} \frac{1}{|\omega-\zeta|^{2}} \frac{d \omega}{\omega-w} .
$$

Let $E^{\prime}=C-\bar{\Omega}^{\prime}$, then according to Green's formula,

$$
f(z)=\frac{1}{\pi} \int_{E^{\prime}} \frac{1}{\overline{(\omega-\zeta)^{2}}} \frac{d \sigma(\omega)}{(\omega-\zeta)(\omega-w)} .
$$

Hence

$$
\begin{aligned}
\Phi(z) & =\frac{w-\zeta}{\pi} \int_{E^{\prime}} \frac{d \sigma(\omega)}{\overline{(\omega-\zeta)^{2}(\omega-\zeta)(\omega-w)}} \\
& =\frac{w-\zeta}{\pi} \int_{E^{\prime}} \frac{d \sigma(\omega)}{\overline{(\omega-\zeta)^{2}}(\omega-\zeta)\left[1-\frac{w-\zeta}{\omega-\zeta}\right]} \\
& =\frac{w-\zeta}{\pi} \int_{E} \frac{d \sigma(t)}{1-t(w-\zeta)}=\frac{1}{\pi} \int_{E} \frac{d \sigma(t)}{\Psi(z)-t} .
\end{aligned}
$$

This concludes the proof.

REMARK. The theorem remains valid for the general case that $\Omega \notin 0_{A D}$. This can be accomplished via a canonical exhaustion of $\Omega$ by regular regions $\left\{\Omega_{m}\right\}$. 
The following corollary was also obtained in Burbea [3] and Sakai [8]. The methods in [8] are different from ours.

CoRollary 1. Let $\Omega \notin 0_{A D}$. Then $\|\Phi\|_{\infty} \leqq C_{D}(\zeta)$ with equality holding if and only if $\Omega$ is conformally equivalent to the unit disk $\triangle$ less (possibly) a closed null $C_{D^{-}}$-set.

Proof. According to Lemma 1 and (3.1) we have

$$
\|\Phi\|_{\infty} \leqq \frac{1}{\pi} \sqrt{\pi \sigma(E)}=C_{D}(\zeta) .
$$

The statement about equality follows from Lemma 1 too.

The theorem of Ahlfors and Beurling [1] is also a consequence of the theorem as the following corollary shows.

CoRollary 2. $C_{D}(\zeta) \leqq C_{B}(\zeta)$.

Proof. We may assume that $C_{D}(\zeta)>0$. Let $f(z)=\Phi(z) / C_{D}(\zeta)$. Since $\Phi(\zeta)=0$ it follows from Corollary 1 that $f \in \mathscr{B}_{\zeta}(\Omega)$. Thus $\left|\Phi^{\prime}(\zeta)\right| / C_{D}(\zeta) \leqq C_{B}(\zeta)$. However, $\Phi^{\prime}(\zeta)=(1 / 2)(a-b)$ and the assertion follows by appealing to (3.1).

REMARK. One can show (see [8]) that equality in the last corollary occurs if and only if either (i) $C_{B}(\zeta)=0$, or (ii) $\Omega$ is conformally equivalent to the unit disk $\Delta$ less (possibly) a closed null $C_{B}$-set.

4. The Schwarzian derivative. We again assume that $\Omega$ is a regular analytic region as mentioned before. The more general case can be always obtained by a canonical exhaustion. Let $H_{S}(\Omega)$ be the Hilbert space of all holomorphic functions $f$ in $\Omega$, having single valued integrals and so that

$$
\|f\|^{2}=\int_{\Omega}|f(z)|^{2} d \sigma(z)<\infty .
$$

This space possesses the (reduced) Bergman kernel function $K_{2 \Omega}(z, \bar{\zeta})$. We have the obvious identity

$$
K_{\Omega}(z, \bar{\zeta})=\frac{1}{\pi} \Phi^{\prime}(z: \zeta)
$$

and therefore

$$
C_{D}(\zeta: \Omega)=\sqrt{\pi K_{\Omega}(\zeta, \bar{\zeta})}
$$


The "adjoint" kernel [2] is given by

$$
L_{\Omega}(z, \zeta)=-\frac{1}{\pi} \Psi^{\prime}(z: \zeta)
$$

This kernel is symmetric in $z$ and $\zeta$. Since $d \Phi=d \bar{\Psi}$ on the boundary, we have $\overline{K_{\Omega}(z, \zeta) d z}=-L_{\varepsilon}(z, \zeta) d z$ for $z \in \partial \Omega$ and $\zeta \in \Omega$. Also,

$$
L_{\Omega}(z, \zeta)=\frac{1}{\pi} \frac{1}{(z-\zeta)^{2}}-l_{\Omega}(z, \zeta),
$$

where $l_{\Omega}(z, \zeta)$ is symmetric and holomorphic in $(z, \zeta) \in \bar{\Omega} \times \bar{\Omega}$. If $\zeta \in \Omega$ is fixed then $l_{\Omega}(, \zeta) \in H_{S}(\Omega)$. We have (see also [2, p. 243])

$$
\left\|l_{\Omega}(, \zeta)\right\|^{2}=\int_{\Omega}\left|l_{\hbar}(z, \zeta)\right|^{2} d \sigma(z)=K_{\Omega}(\zeta, \bar{\zeta})-\Gamma_{\Omega}(\zeta, \bar{\zeta})
$$

where

$$
\Gamma_{\Omega}(\zeta, \bar{\zeta})=\frac{1}{\pi^{2}} \int_{E_{\Omega}} \frac{d \sigma(z)}{|z-\zeta|^{4}} .
$$

Here $E_{\Omega}=\hat{\boldsymbol{C}}-\bar{\Omega}$. We also write

$$
\alpha(\zeta)=\alpha(\zeta: \Omega)=\sqrt{\pi \Gamma_{\Omega}(\zeta, \bar{\zeta})}
$$

and thus $\pi \alpha^{2}(\zeta)$ represents the image area of $E_{3}$ under the linear mapping $(z-\zeta)^{-1}$. Therefore

$$
\alpha(\zeta) \leqq \sqrt{\pi K_{\Omega}(\zeta, \bar{\zeta})}=C_{D}(\zeta: \Omega),
$$

equality holding, for each $\zeta \in \Omega$, only if $\partial \Omega$ is a circle (including circles passing through $\infty$ ). Further, we have

$$
l_{\Omega}(z, \zeta)=\left(l_{\Omega}(, \zeta), K_{\Omega}(, \bar{z})\right)
$$

and therefore

$$
\left|l_{\Omega}(z, \zeta)\right|^{2} \leqq\left\|l_{\Omega}(, \zeta)\right\|^{2}\left\|K_{\Omega}(, \bar{z})\right\|^{2}
$$

or

$$
\left|l_{\Omega}(z, \zeta)\right|^{2} \leqq K_{\Omega}(z, \bar{z})\left[K_{\Omega}(\zeta, \bar{\zeta})-\Gamma_{\Omega}(\zeta, \bar{\zeta})\right]
$$

A fortiori,

$$
\left|l_{\Omega}(z, \zeta)\right|^{2} \leqq K_{\Omega}(z, \bar{z}) K_{\Omega}(\zeta, \bar{\zeta}) .
$$

Let $\omega=\phi(z)$ be a conformal mapping of $\Omega$ onto $\Omega^{*}$. Then, for $\tau=\phi(\zeta)$,

$$
K_{\Omega}(z, \bar{\zeta})=K_{\Omega^{*}}(\omega, \bar{\tau}) \phi^{\prime}(z) \overline{\phi^{\prime}(\zeta)}
$$


and

$$
L_{\Omega}(z, \zeta)=L_{\Omega^{*}}(\omega, \tau) \phi^{\prime}(z) \phi^{\prime}(\zeta) .
$$

From the last formula it follows that

$$
l_{\Omega^{*}}(\omega, \tau) \phi^{\prime}(z) \phi^{\prime}(\zeta)=l_{\Omega}(z, \zeta)-\frac{1}{6 \pi} S_{\phi}(z, \zeta),
$$

where

$$
S_{\phi}(z, \zeta)=-6 \frac{\partial^{2}}{\partial z \partial \zeta} \log \frac{\phi(z)-\phi(\zeta)}{z-\zeta}
$$

we note that

$$
S_{\phi}(z, z)=S_{\phi}(z)=\left(\frac{\phi^{\prime \prime}}{\phi^{\prime}}\right)^{\prime}-\frac{1}{2}\left(\frac{\phi^{\prime \prime}}{\phi^{\prime}}\right)^{2} ; \quad \phi=\phi(z), z \in \Omega,
$$

is the Schwarzian derivative of $\phi(z)$.

From (4.4) we have

$$
\left|l_{\Omega^{*}}(\omega, \tau)\right|^{2} \leqq K_{\Omega^{*}}(\omega, \bar{\omega}) K_{\Omega^{*}}(\tau, \bar{\tau}),
$$

and therefore, using (4.5) and (4.6),

$$
\left|l_{\Omega}(z, \zeta)-\frac{1}{6 \pi} S_{\phi}(z, \zeta)\right|^{2} \leqq K_{\Omega}(z, \bar{z}) K_{\Omega}(\zeta, \bar{\zeta}) .
$$

Consequently,

$$
\left|S_{\phi}(z, \zeta)\right| \leqq 6 \pi\left\{\left[K_{\Omega}(z, \bar{z}) K_{\Omega}(\zeta, \bar{\zeta})\right]^{1 / 2}+\left|l_{\Omega}(z, \zeta)\right|\right\} .
$$

In view of (4.1), (4.2) and (4.3) we therefore have

$$
\left|S_{\phi}(z, \zeta)\right| \leqq 6 C_{D}(z) C_{D}(\zeta)\left[1+\left(1-\frac{\alpha^{2}(\zeta)}{C_{D}^{2}(\zeta)}\right)^{1 / 2}\right] .
$$

This is the desired result. If now we use (2.2), we arrive at our main theorem:

THEOREM 2. Let $\Omega \notin 0_{A D}$. If $\phi$ is holomorphic and univalent in $\Omega$ we have the following sharp string of inequalities

$$
\begin{aligned}
\left|S_{\phi}(z, \zeta)\right| & \leqq 6 C_{D}(z) C_{D}(\zeta)\left[1+\left(1-\frac{\alpha^{2}(\zeta)}{C_{D}^{2}(\zeta)}\right)^{1 / 2}\right] \\
& \leqq 6 C_{B}(z) C_{B}(\zeta)\left[1+\left(1-\frac{\alpha^{2}(\zeta)}{C_{B}^{2}(\zeta)}\right)^{1 / 2}\right] \\
& \leqq 6 C_{\beta}(z) C_{\beta}(\zeta)\left[1+\left(1-\frac{\alpha^{2}(\zeta)}{C_{\beta}^{2}(\zeta)}\right)^{1 / 2}\right]
\end{aligned}
$$




$$
\leqq 6 \lambda_{\Omega}(z) \lambda_{\Omega}(\zeta)\left[1+\left(1-\frac{\alpha^{2}(\zeta)}{\lambda_{\Omega}^{2}(\zeta)}\right)^{1 / 2}\right]
$$

Proof. The above holds for regular regions. The general case is obtained by a canonical exhaustion.

CoROLlaRY 3. Let $\Omega \notin 0_{G}$ and let $\phi$ be holomorphic and univalent in $\Omega$. Then

$$
\left|S_{\phi}(z, \zeta)\right| \leqq 6 \lambda_{\Omega}(z) \lambda_{\Omega}(\zeta)\left[1+\left(1-\frac{\alpha^{2}(\zeta)}{\lambda_{\Omega}^{2}(\zeta)}\right)^{1 / 2}\right] ; \quad z, \zeta \in \Omega,
$$

and in particular

$$
\left|S_{\varphi}(z)\right| \leqq 6 \lambda_{\Omega}^{2}(z)\left[1+\left(1-\frac{\alpha^{2}(z)}{\lambda_{\Omega}^{2}(z)}\right)^{1 / 2}\right] ; \quad z \in \Omega .
$$

The inequalities are sharp. The inequality

$$
\left|S_{\varphi}(z)\right| \leqq 6 \lambda_{\Omega}^{2}(z)
$$

is sharp only when $\Omega$ is a disk less (possibly) a closed subset of innear capacity zero. Otherwise, we have the sharp inequality

$$
\left|S_{\varphi}(z)\right| \leqq 12 \lambda_{2}^{2}(z)
$$

Proof. This follows from the fact that $\alpha(z)=\alpha(z: \Omega) \leqq \lambda_{\Omega}(z)$ and equality at all points $z \in \Omega$ holds if and only if $\Omega$ is a disk less (possibly) a closed subsets of inner capacity zero.

This generalizes the contents of Propositions 1 and 2.

Added in proof. The author has learned A. F. Beardon and F. W. Gehring have recently also generalized the contents of the present Proposition 2.

\section{REFERENCES}

1. L. Ahlfors and A. Beurling, Conformal invariants and function-theoretic null sets, Acta Math., 83 (1950), 101-129.

2. S. Bergman and M. Schiffer, Kernel functions and conformal mapping, Compositio Math., 8 (1951), 205-249.

3. J. Burbea, Capacities and spans on Riemann surfaces, Proc. Amer. Math. Soc., 72 (1979), 327-332.

4. F.W. Gehring, Univalent functions and the Schwarzian derivative, Comment. Math. Helvetici, 52 (1977), 561-572.

5. W. Kraus, Über den Zusammenhang einiger Charakteristiken eines einfach zusammenhängenden Bereiches mit der Kreisabbildung, Mitt. Math. Sem. Giessen, 21 (1932), $1-28$,

6. O. Lehto, Quasiconformal Mappings in the Plane, Lecture Notes 14, University of Maryland, 1975. 
7. Z. Nehari, The Schwarzian derivative and Schlicht functions, Bull. Amer. Math. Soc., 55 (1949), 545-551.

8. M. Sakai, Analytic functions with finite Dirichlet integrals on Riemann surfaces, Acta Math., 142 (1979), 199-220.

9. M. Schiffer, The span of multiply connected domains, Duke Math. J., 10 (1943), 209-216.

Received October 31, 1978 and in revised form March 19, 1979.

UNIVERSITY OF PittsBuRgh

PitTSBURGh, PA 15260 


\section{PACIFIC JOURNAL OF MATHEMATICS}

\section{EDITORS}

DONALD BABBITT (Managing Editor)

University of California

Los Angeles, CA 90024

HUgo RossI

University of Utah

Salt Lake City, UT 84112

C. C. Moore and ANDrew OGG

University of California

Berkeley, CA 94720

\section{J. DUGUNDJI}

Department of Mathematics

University of Southern California

Los Angeles, CA 90007

R. FinN and J. MILGRaM

Stanford University

Stanford, CA 94305

\section{ASSOCIATE EDITORS}
E. F. BECKENBACH
B. H. NeumanN
F. WOLF
K. YOSHIDA

\section{SUPPORTING INSTITUTIONS}

UNIVERSITY OF BRITISH COLUMBIA CALIFORNIA INSTITUTE OF TECHNOLOGY

UNIVERSITY OF CALIFORNIA

MONTANA STATE UNIVERSITY

UNIVERSITY OF NEVADA, RENO

NEW MEXICO STATE UNIVERSITY

OREGON STATE UNIVERSITY

UNIVERSITY OF OREGON
UNIVERSITY OF SOUTHERN CALIFORNIA

STANFORD UNIVERSITY

UNIVERSITY OF HAWAII

UNIVERSITY OF TOKYO

UNIVERSITY OF UTAH

WASHINGTON STATE UNIVERSITY

UNIVERSITY OF WASHINGTON

The Supporting Institutions listed above contribute to the cost of publication of this Journal, but they are not owners or publishers and have no responsibility for its content or policies.

Mathematical papers intended for publication in the Pacific Journal of Mathematics should be in typed form or offset-reproduced, (not dittoed), double spaced with large margins. Please do not use built up fractions in the text of the manuscript. However, you may use them in the displayed equations. Underline Greek letters in red, German in green, and script in blue. The first paragraph or two must be capable of being used separately as a synopsis of the entire paper. Please propose a heading for the odd numbered pages of less than 35 characters. Manuscripts, in triplicate, may be sent to any one of the editors. Please classify according to the scheme of Math. Reviews, Index to Vol. 39. Supply name and address of author to whom proofs should be sent. All other communications should be addressed to the managing editor, or Elaine Barth, University of California, Los Angeles, California, 90024.

50 reprints to each author are provided free for each article, only if page charges have been substantially paid. Additional copies may be obtained at cost in multiples of 50 .

The Pacific Journal of Mathematics is issued monthly as of January 1966. Regular subscription rate: $\$ 84.00$ a year (6 Vols., 12 issues). Special rate: $\$ 42.00$ a year to individual members of supporting institutions.

Subscriptions, orders for numbers issued in the last three calendar years, and changes of address should be sent to Pacific Journal of Mathematics, P.O. Box 969, Carmel Valley, CA 93924, U.S.A. Older back numbers obtainable from Kraus Periodicals Co., Route 100, Millwood, NY 10546.

PUBLISHED BY PACIFIC JOURNAL OF MATHEMATICS, A NON-PROFIT CORPORATION

Printed at Kokusai Bunken Insatsusha (International Academic Printing Co., Ltd.). 8-8, 3-chome, Takadanobaba, Shinjuku-ku, Tokyo 160, Japan.

Copyright (C) 1979 by Pacific Journal of Mathematics Manufactured and first issued in Japan 


\section{Pacific Journal of Mathematics}

\section{Vol. 85, No. $2 \quad$ October, 1979}

Charles A. Akemann and Steve Wright, Compact and weakly compact derivations of $C^{*}$-algebras ........................... 253

Dwight Richard Bean, Andrzej Ehrenfeucht and George Frank McNulty, Avoidable patterns in strings of symbols ................... 261

Richard Clark Brown, Notes on generalized boundary value problems in Banach spaces. I. Adjoint and extension theory.................

Kenneth Alexander Brown and John William Lawrence, Injective hulls of group rings .................................... 323

Jacob Burbea, The Schwarzian derivative and the Poincaré metric ....... 345

Stefan Andrus Burr, On the completeness of sequences of perturbed polynomial values ....................................

Peter H. Chang, On the characterizations of the breakdown points of quasilinear wave equations..............................

Joseph Nicholas Fadyn, The projectivity of $\operatorname{Ext}(T, A)$ as a module over

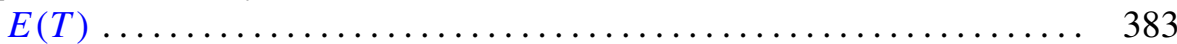

Donald Eugene Maurer, Arithmetic properties of the idèle discriminant .... 393

Stuart Rankin, Clive Reis and Gabriel Thierrin, Right subdirectly irreducible semigroups ................................. 403

David Lee Rector, Homotopy theory of rigid profinite spaces. I ........ 413 Raymond Moos Redheffer and Wolfgang V. Walter, Comparison theorems for parabolic functional inequalities................

H. M. (Hari Mohan) Srivastava, Some generalizations of Carlitz's theorem

James Alan Wood, Unbounded multipliers on commutative Banach algebras....

T. Yoshimoto, Vector-valued ergodic theorems for operators satisfying norm

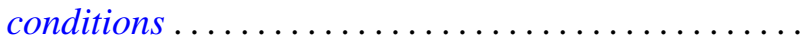

Jerry Searcy and B. Andreas Troesch, Correction to: "A cyclic inequality and a related eigenvalue problem".

Leslie Wilson, Corrections to: "Nonopenness of the set of Thom-Boardman maps" 